\title{
Monte Carlo modelling of Th-Pb fuel assembly with californium neutron source
}

\author{
Mikołaj Oettingen, \\ Przemystaw Stanisz
}

\begin{abstract}
This paper describes the methodology developed for the numerical reconstruction and modelling of the thorium-lead (Th-Pb) assembly available at the Department of Nuclear Energy, Faculty of Energy and Fuels, AGH University, Krakow, Poland. This numerical study is the first step towards integral irradiation experiments in the Th-Pb environment. The continuous-energy Monte Carlo burnup (MCB) code available on supercomputer Prometheus of ACK Cyfronet AGH was applied for numerical modelling. The assembly consists of a hexagonal array of $\mathrm{ThO}_{2}$ fuel rods and metallic $\mathrm{Pb}$ rods. The design allows for different arrangements of the rods for various types of irradiations and experimental measurements. The intensity of the fresh neutron source intended for integral experiments is about $10^{8} \mathrm{n} / \mathrm{s}$, which corresponds to the mass of about $43 \mu \mathrm{g}{ }^{252} \mathrm{Cf}$. The source was modelled in the form of $\mathrm{Cf}_{2} \mathrm{O}_{3}$-Pd cermet wire embedded in two stainless steel capsules.
\end{abstract}

Keywords: californium $\bullet$ lead $\bullet$ Monte Carlo $\bullet$ MCB $\bullet$ thorium

M. Oettingen ${ }^{\bowtie}$, P. Stanisz

Department of Nuclear Energy

Faculty of Energy and Fuels

AGH University of Science and Technology

30 Mickiewicza Av., 30-059 Krakow, Poland

E-mail: moettin@agh.edu.pl

Received: 2 January 2018

Accepted: 16 July 2018

\section{Introduction}

Current light-water nuclear reactors operate on the enriched uranium fuel. The main fissile isotope in such fuel is ${ }^{235} \mathrm{U}$; however, in a series of transmutations and decays, fissile isotopes of plutonium (i.e. ${ }^{239} \mathrm{Pu}$ and ${ }^{241} \mathrm{Pu}$ ) are formed. In general, about $70 \%$ of fissions during a reactor cycle in commercial nuclear reactors occur on ${ }^{235} \mathrm{U}$, whereas about $30 \%$ of fissions occur on ${ }^{239} \mathrm{Pu}$ and ${ }^{241} \mathrm{Pu}$. For this reason, the process of uranium fissioning and plutonium breeding, as well as further in situ fissioning in the reactor core, is called the uranium-plutonium (U-Pu) fuel cycle. An alternative to this is the thorium-uranium (Th-U) fuel cycle. Thorium $\left({ }^{232} \mathrm{Th}\right)$ itself cannot undergo nuclear fission but serves as a fertile isotope for the production of fissile ${ }^{233} \mathrm{U}$. Therefore, it is mixed with fissile isotopes such as ${ }^{233} \mathrm{U},{ }^{235} \mathrm{U}$ or ${ }^{239} \mathrm{Pu}$ in order to provide surplus neutrons for the breeding of ${ }^{233} \mathrm{U}$. The natural abundance of thorium is about three times larger than that of uranium. In addition, spent nuclear fuel from thorium contains less minor actinides and plutonium. Furthermore, it is contaminated with easily detectable ${ }^{232} \mathrm{U}$, which is a big advantage for non-proliferation $[1,2]$. However, the only country that conducts advanced research on the thorium fuel cycle is India, due to the large resources of thorium and limited resources of uranium [3]. Thus, the knowledge about the behaviour of thorium-based fuels under irradiation is still limited. In this paper, we present the results of numerical simulations of the $\mathrm{Th}-\mathrm{Pb}$ fuel assembly with $\mathrm{Cf}$ neutron source. The numerical analysis 
precedes the campaign of irradiation experiments planned with the available nuclear set-up. The following scientific topics were taken into account during the simulations:

- adjustment of configuration for thermal and fast irradiations,

- estimation of the irradiation environment i.e. neutron spectra in both configurations, and

- modelling of thorium fuel sample irradiation in both configurations.

Next section "Th-Pb fuel assembly" describes the available nuclear set-up and its numerical model developed using the Monte Carlo burnup (MCB) code. Section "Results" shows the results of numerical simulations. The study is concluded in the last section "Discussion and conclusions".

\section{Th- $\mathrm{Pb}$ fuel assembly}

The Th- $\mathrm{Pb}$ fuel assembly located in the radiometric laboratory of the AGH University consists of three main components: the support structure, the lead reflector and the core. Cylindrical top plate, top bars and grid determine the rectangular shape of the assembly core. The side bars connect the top and the bottom support plates to provide stability. The structural elements were fabricated at the
AGH University using standard construction steel. The $\mathrm{Pb}$ reflector surrounds the assembly core. The reflector was manufactured using rectangular $\mathrm{Pb}$ bricks $(10 \times 10 \times 5 \mathrm{~cm})$ specially designed for the radiation shielding purposes. The core consists of $\mathrm{Pb}$ and $\mathrm{ThO}_{2}$ rods arranged in a hexagonal pattern. The height of the core equals $100 \mathrm{~cm}$, while the rod pitch is $1.3 \mathrm{~cm}$ high [4]. The hexagonal shape of the $\mathrm{Pb}$ rods allows for their compact package, while the cylindrical shape of the $\mathrm{ThO}_{2}$ rods enables easy replacement of $\mathrm{Pb}$ rods without dismantling the whole assembly. These features of the assembly allow for easy rearrangement of the core pattern in many types of irradiation experiments and for the insertion of additional elements, such as polyethylene rods for neutron thermalizations. The $\mathrm{ThO}_{2}$ fuel rods were manufactured in the Bhabha Atomic Research Centre (BARC) [5] of India, while the metallic $\mathrm{Pb}$ rods were made at the AGH University. Both types of rods were equipped with pins on both ends for their precise placement in the bottom plate and $\mathrm{Al}$ fixing bars. In the current initial pattern presented in Fig. 1, seven innermost $\mathrm{ThO}_{2}$ rods were removed from the assembly. Six of them were replaced by $\mathrm{Pb}$ rods for the intensification of the neutron flux due to $(n, 2 n)$ reactions on lead. The free space remaining after reloading of the most central rod serves as an insertion hole for $\mathrm{Cf}$ neutron source.
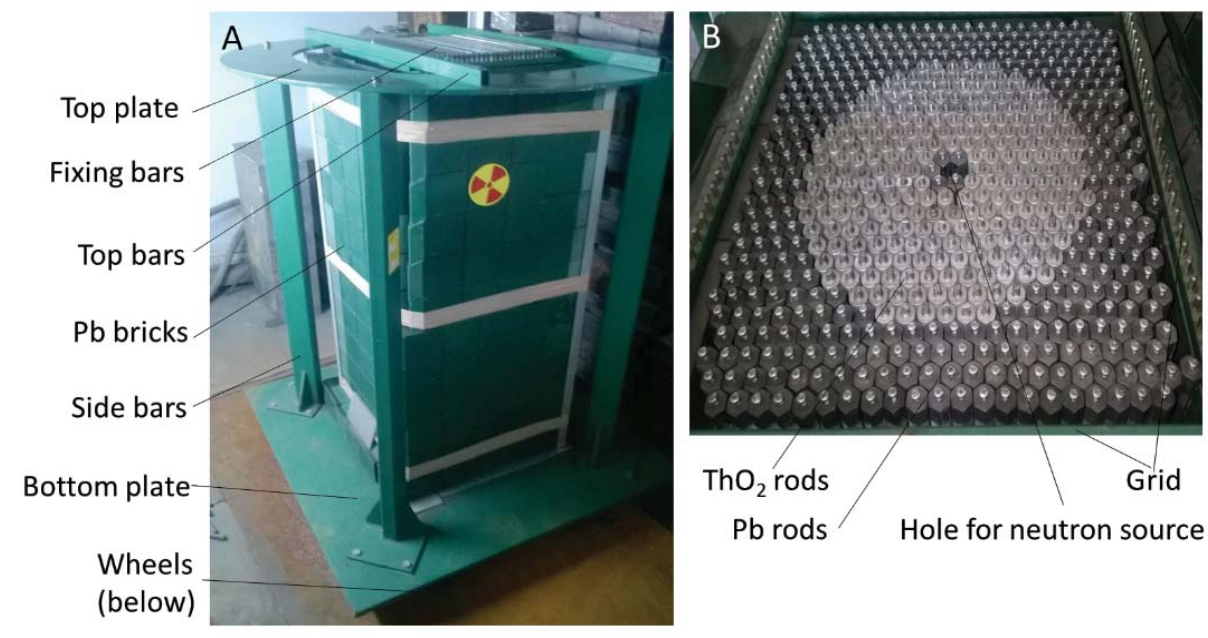

Fig. 1. Th- $\mathrm{Pb}$ fuel assembly.
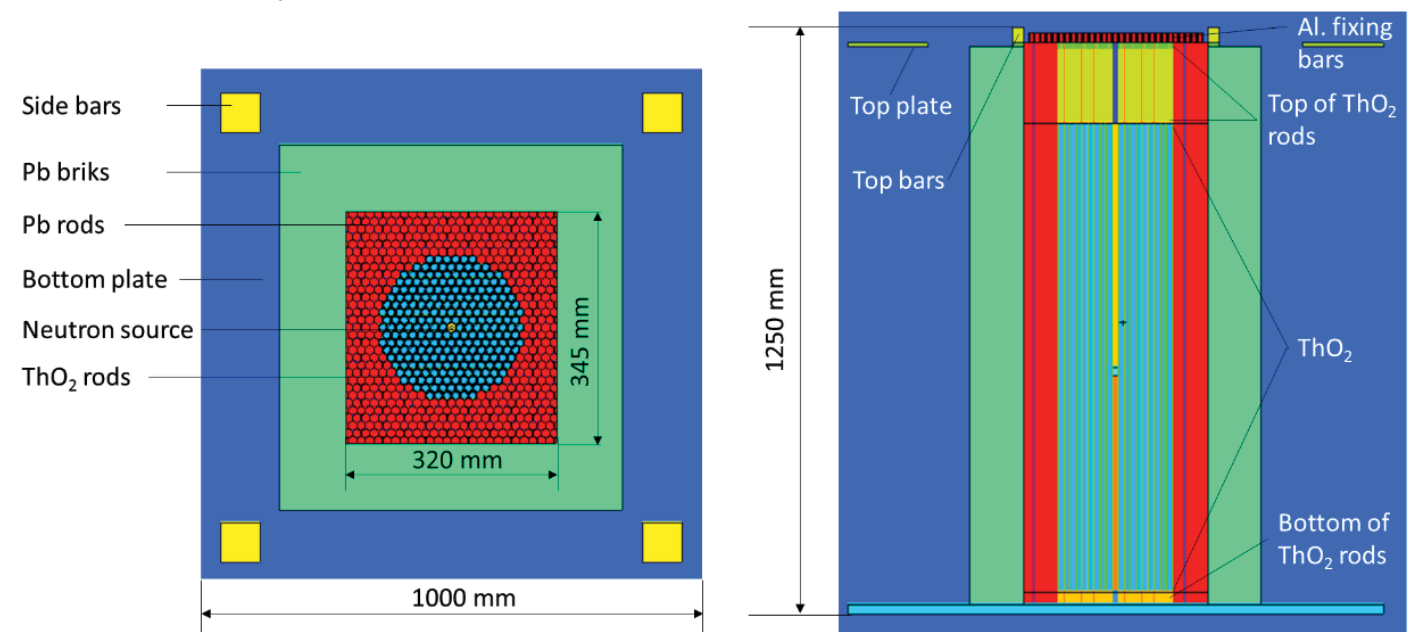

Fig. 2. Cross-sections of the numerical model. 
The continuous-energy MCB code developed at the AGH University, Faculty of Energy and Fuels, Department of Nuclear Energy, was used for all numerical simulations [6]. The MCB code integrates the commercial Monte Carlo transport code - general Monte Carlo N-particle transport (MCNP) code [7] - and the novel transmutation trajectory analysis (TTA) code [8] at the level of the FORTRAN source code. The MCNP subroutines use Monte Carlo methods for neutron transport simulations, while the TTA forms and analyses transmutation and decay chains for nuclide density evolution in time function. In the present calculations, the ENDF/B-VII nuclear data libraries for the temperature of $300 \mathrm{~K}$ were applied. Figure 2 shows the cross-sections of the assembly core with its surroundings. Some simplifications were introduced to the numerical geometry of the Th rods. The rods were divided into three sections representing the top of the rod, $\mathrm{ThO}_{2}$ pellets and the bottom of the rod. The $\mathrm{ThO}_{2}$ in the inner section was not divided into particular fuel pellets, but it was modelled as a one cylinder with the height of $100 \mathrm{~cm}$. The introduced simplifications do not distort the numerical results but significantly reduce the complexity of the model. The outer $\mathrm{Pb}$ neutron reflector was modelled as a compact cell surrounding the assembly core. The materials used for the numerical reconstruction of the assembly are presented in Table 1 .

\section{Results}

The numerical simulations were performed for $10^{6}$ neutrons per six time steps of five days each, which gives 30 days of irradiation in total. The precision

Table 1. Materials composing the numerical model $[9,10]$

\begin{tabular}{|c|c|c|}
\hline No. & Material & $\begin{array}{l}\text { Density } \\
{\left[\mathrm{g} / \mathrm{cm}^{3}\right]}\end{array}$ \\
\hline 1 & $\mathrm{ThO}_{2}$ pellets & 9.4 \\
\hline 2 & $\mathrm{Cf}_{2} \mathrm{O}_{3}-\mathrm{Pd}$ source & 12.023 \\
\hline 3 & $\mathrm{~Pb}$ reflector & 11.35 \\
\hline 4 & SS304L source capsules & 8.0 \\
\hline 5 & Aluminium fixing bars, plugs, clad & 2.7 \\
\hline 6 & Steel plates & 7.85 \\
\hline 7 & Air free spaces & 0.001205 \\
\hline No. & $\begin{array}{l}\text { Homogenized materials } \\
\text { (by volume) }\end{array}$ & $\begin{array}{l}\text { Density } \\
{\left[\mathrm{g} / \mathrm{cm}^{3}\right]}\end{array}$ \\
\hline 1 & Steel $52 \%+$ air $48 \%$ grid & 4.08 \\
\hline 2 & Steel $31 \%$ + air $69 \%$ side bars & 2.40 \\
\hline 3 & Steel $13 \%+$ air $87 \%$ top bars & 1.01 \\
\hline 4 & Inconel 5\% + air 95\% spring & 0.4246 \\
\hline No. & $\mathrm{ThO}_{2}$ rod & \\
\hline 1 & Mass of Th & $\sim 736 \mathrm{~g}$ \\
\hline 2 & Mass of $\mathrm{ThO}_{2}$ & $\sim 836 \mathrm{~g}$ \\
\hline 3 & Number of pellets & 100 \\
\hline 4 & Pellet length & $10 \mathrm{~mm}$ \\
\hline 5 & Pellet diameter & $1.064 \mathrm{~mm}$ \\
\hline 6 & Gap thickness & $0.13 \mathrm{~mm}$ \\
\hline 7 & Clad thickness & $0.85 \mathrm{~mm}$ \\
\hline
\end{tabular}

(relative error) for all calculations is $<0.1$ and in most cases, <0.05, which corresponds to generally reliable results [7]. The supercomputer Prometheus of the ACK Cyfronet AGH was used for all simulations [11]. The intensity of the fresh Cf source equals $10^{8} \mathrm{n} / \mathrm{s}$, which corresponds to the mass of about $43 \mu \mathrm{g}{ }^{252} \mathrm{Cf}$. The source was modelled in the form of $\mathrm{Cf}_{2} \mathrm{O}_{3}-\mathrm{Pd}$ cermet wire embedded in two stainless steel capsules and placed in the middle of the assembly [12]. The neutron spectrum of the source was adjusted using inherent features of the MCB code [5]. However, irradiations with other neutron sources, such as D-T, could also be investigated [13]. In the analysis, we considered two different configurations of the Th- $\mathrm{Pb}$ assembly: fast spectrum configuration with a thorium core and shielding lead rods and thermal configuration with additional layers of polyethylene for spectrum thermalization.

In the first step of the calculations, we adjusted the optimal configuration of the assembly for irradiations in the fast neutron spectrum. We took into consideration the absolute value of the neutron flux as well as the neutron spectrum in 100 energy groups as the crucial parameters determining the irradiation environment. Figure 3 shows neutron spectra for fast configuration of the assembly for eight radial positions (R1-R8) corresponding to eight irradiation zones. R1 is the innermost position, while R8 is the outermost position. The spectra were calculated in the free space between the rods filled with only air and intended for placement of the material sample. The volume of the space equals $0.35 \mathrm{~cm}^{3}$. The space is located vertically just in the middle of the Th fuel column, at the same level as Cf neutron sources. As it is shown, the spectra are characterized only by the fast energy component. The thermal component is negligible, as presented in Table 2. The maximum value of the fast flux occurs just at the neutron source in the zone R1, which is consistent with theoretical prediction. Figure 4 shows a drop of the neutron flux towards the outer zones of the assembly. The most significant drop occurs between the zones R1 and R2 - the flux falls about three times. Considering the aforementioned justification, for the subsequent analysis, we chose the zone R1 as the best place for fast irradiations.

In order to adjust the optimal thermal irradiation position, thermalization zones made of hexagonal polyethylene rods $\left(\mathrm{C}_{2} \mathrm{H}_{4}\right)$ with a density of $0.92 \mathrm{~g} / \mathrm{cm}^{3}$ were introduced to the assembly. We chose polyethylene because of its good moderation capabilities resulting from the large content of hy-

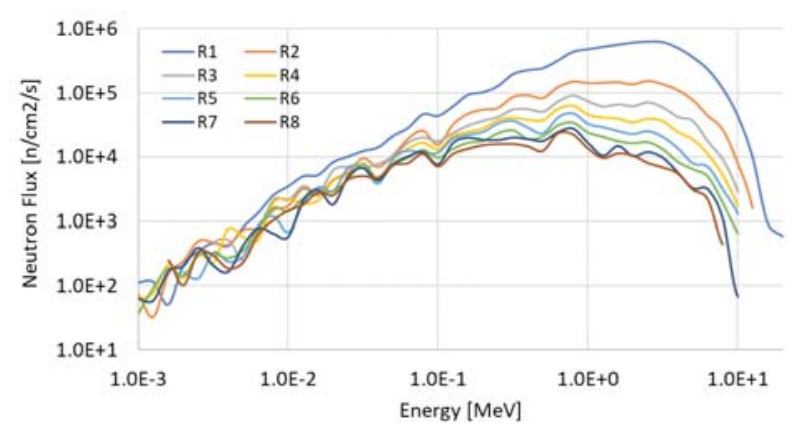

Fig. 3. Neutron spectra for fast configuration. 
Table 2. Absolute neutron fluxes

\begin{tabular}{|c|c|c|c|c|}
\hline \multicolumn{5}{|c|}{ Fast configuration flux $\left[\mathrm{n} / \mathrm{cm}^{2} / \mathrm{s}\right]$} \\
\hline$\overline{\text { Zone }}$ & R1 & $\mathrm{R} 2$ & R3 & $\mathrm{R} 4$ \\
\hline Placement* $[\mathrm{cm}]$ & 1.3 & 2.6 & 3.9 & 5.2 \\
\hline$\phi_{t h 1}(\leq 1 \mathrm{eV})$ & $0.0 \mathrm{E}+00$ & $0.0 \mathrm{E}+00$ & $0.0 \mathrm{E}+00$ & $0.0 \mathrm{E}+00$ \\
\hline$\phi_{r}-\phi_{t h 2}(\leq 1 \mathrm{keV})$ & $3.8 \mathrm{E}+02$ & $7.3 \mathrm{E}+01$ & $3.2 \mathrm{E}+02$ & $1.8 \mathrm{E}+02$ \\
\hline$\phi_{t}-\phi_{f}(<20 \mathrm{MeV})$ & $6.6 \mathrm{E}+06$ & $2.0 \mathrm{E}+06$ & $1.1 \mathrm{E}+06$ & $7.3 \mathrm{E}+05$ \\
\hline Zone & R5 & R6 & $\mathrm{R} 7$ & $\mathrm{R} 8$ \\
\hline Placement* $[\mathrm{cm}]$ & 6.5 & 7.8 & 9.1 & 10.4 \\
\hline$\phi_{t h 1}(\leq 1 \mathrm{eV})$ & $0.0 \mathrm{E}+00$ & $0.0 \mathrm{E}+00$ & $0.0 \mathrm{E}+00$ & $0.0 \mathrm{E}+00$ \\
\hline$\phi_{r}-\phi_{\text {th } 2}(\leq 1 \mathrm{keV})$ & $2.1 \mathrm{E}+02$ & $1.9 \mathrm{E}+02$ & $8.8 \mathrm{E}+01$ & $1.7 \mathrm{E}+02$ \\
\hline$\phi_{t}-\phi_{f}(<20 \mathrm{MeV})$ & $5.4 \mathrm{E}+05$ & $4.1 \mathrm{E}+05$ & $3.3 \mathrm{E}+05$ & $2.7 \mathrm{E}+05$ \\
\hline \multicolumn{5}{|c|}{ Thermal configuration flux $\left[\mathrm{n} / \mathrm{cm}^{2} / \mathrm{s}\right]$} \\
\hline Zone & R2 & R3 & R4 & R5 \\
\hline$\phi_{t h 1}(\leq 1 \mathrm{eV})$ & $3.2 \mathrm{E}+04$ & $9.6 \mathrm{E}+04$ & $1.0 \mathrm{E}+05$ & $8.9 E+04$ \\
\hline$\phi_{r}(>1 \mathrm{eV} \wedge \leq 1 \mathrm{keV})$ & $1.1 \mathrm{E}+05$ & $1.3 \mathrm{E}+05$ & $1.0 \mathrm{E}+05$ & $7.4 \mathrm{E}+04$ \\
\hline$\phi_{t h 2}(\leq 1 \mathrm{keV})$ & $1.4 \mathrm{E}+05$ & $2.3 \mathrm{E}+05$ & $2.0 \mathrm{E}+05$ & $1.6 \mathrm{E}+05$ \\
\hline$\phi_{f}(\geq 1 \mathrm{keV})$ & $1.9 E+06$ & $9.3 \mathrm{E}+05$ & $4.8 \mathrm{E}+05$ & $2.9 \mathrm{E}+05$ \\
\hline$\phi_{t}(<10 \mathrm{MeV})$ & $2.1 \mathrm{E}+06$ & $1.2 \mathrm{E}+06$ & $6.9 \mathrm{E}+05$ & $4.5 \mathrm{E}+05$ \\
\hline
\end{tabular}

*From the centre of Cf neutron source to the centre of the considered zone.

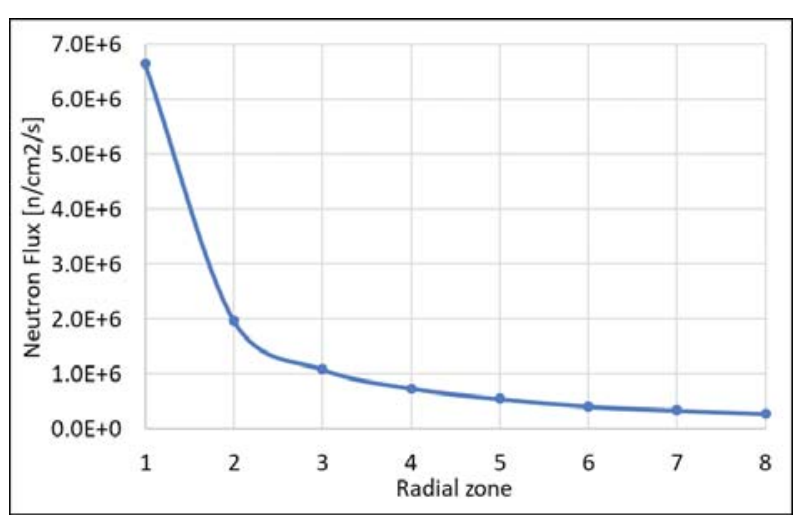

Fig. 4. Drop of absolute flux for fast configuration.

drogen. Moreover, polyethylene rods could be easily and economically manufactured and placed in the assembly. In the analysis, we replaced consequent four inner zones of thorium rods by polyethylene rods and calculated the neutron spectrum and the absolute neutron flux values in the following thorium zone adjacent to the polyethylene zone. The neutron spectra presented in Fig. 5 show fast and thermal energy peaks. It is visible that one zone of $\mathrm{C}_{2} \mathrm{H}_{4}$ is not enough to provide large shift from fast flux to thermal flux. Thus, we introduced additional zones and compared thermal $\phi_{t h}$, resonance $\phi_{r}$

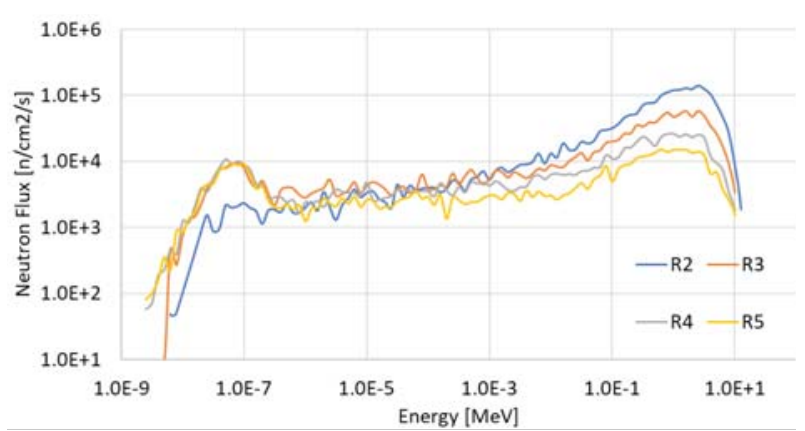

Fig. 5. Neutron spectra for thermal configuration.
$(>1 \mathrm{eV} \leq 1 \mathrm{keV})$, fast $\phi_{f}(\geq 1 \mathrm{kV})$ and total $\phi_{t}$ fluxes in Table 2 . We present two absolute values of thermal fluxes, which depend on the energy groups. The $\phi_{t h 1}$ shows thermal flux with energies $\leq 1 \mathrm{eV}$, while $\phi_{t h 2}$ shows thermal flux with energies $\leq 1 \mathrm{keV}$. The energy group structure of the neutron flux depends on the intended use, e.g. for deterministic calculation tools, the two-group flux structure (thermal and fast) is usually applied. The thermal flux $\phi_{t h i}$ achieves the peak value in the zone $\mathrm{R} 4$ with three layers of $\mathrm{C}_{2} \mathrm{H}_{4}$ rods around the $\mathrm{Cf}$ neutron source, while the thermal flux $\phi_{t h 2}$ achieves the peak value in the zone R3 with two layers of $\mathrm{C}_{2} \mathrm{H}_{4}$ rods. Therefore, the choice of the irradiation position for thermal irradiations depends on the exact description of the experiment and the isotopic composition of the material intended for irradiation.

Finally, we showed microscopic reaction rates and microscopic effective cross-sections of neutron capture and fission for the $\mathrm{ThO}_{2}$ sample placed in the chosen irradiation positions for fast configuration and thermal configuration, respectively. Figure 6 shows neutron spectra in the samples for both irradiations. As it is shown in Table 3, thermal configuration is much better for an experiment on thorium due to its larger capture cross-section in the thermal energy range. The estimated reaction rates may serve to estimate the production rate of ${ }^{233} \mathrm{~Pa}$

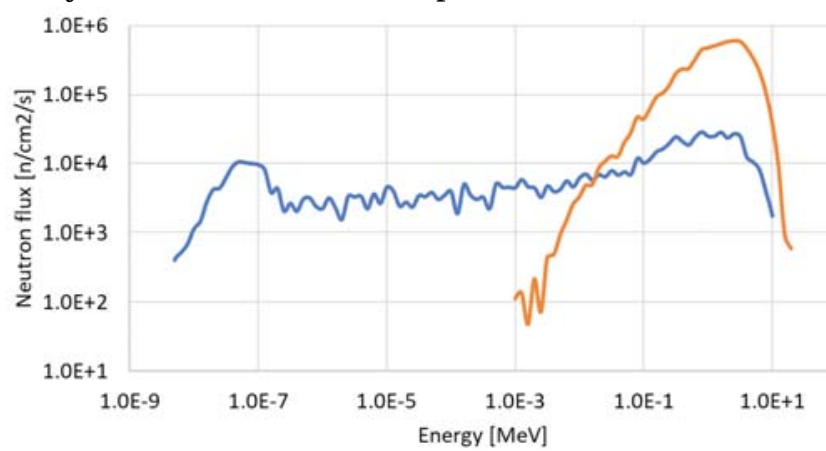

Fig. 6. Neutron spectra in the thorium fuel sample. 
Table 3. Neutronic parameters

\begin{tabular}{lccccc}
\hline \multicolumn{1}{c}{ Position } & $R_{f}(1 / \mathrm{s})$ & $R_{c}(1 / \mathrm{s})$ & $\sigma_{f}^{e f}(\mathrm{~b})$ & $\sigma_{c}{ }^{e f}(\mathrm{~b})$ & $\phi_{t}\left[\mathrm{n} / \mathrm{cm}^{2} / \mathrm{s}\right]$ \\
\hline Fast & $4.58 \mathrm{E}-19$ & $6.76 \mathrm{E}-19$ & 0.07 & 0.10 & $6.53 \mathrm{E}+06$ \\
Thermal & $1.74 \mathrm{E}-20$ & $6.55 \mathrm{E}-18$ & 0.03 & 9.43 & $6.94 \mathrm{E}+05$ \\
\hline
\end{tabular}

and later of fissile ${ }^{233} U[14]$. The neutron interactions with thorium in the fast spectrum are negligible due to its very low capture cross-section; therefore, this position may rather serve for irradiations of isotopes reacting with fast neutrons, such as natural uranium containing ${ }^{238} \mathrm{U}$. This may later serve for the estimation of ${ }^{239} \mathrm{~Np}$ and fissile ${ }^{239} \mathrm{Pu}[15]$.

\section{Discussion and conclusions}

In this paper, we present numerical simulations leading to the estimation of the irradiation conditions in the Th-Pb fuel assembly. The numerical model developed allows for advanced numerical simulations and calculations of a wider range of neutronic parameters towards the research on the thorium fuel cycle using the Th-Pb assembly. In the analysis, we performed a series of numerical simulations towards the estimation of optimal positions for thermal and fast irradiations. The calculated neutron spectra and fluxes show, in a straightforward manner, the optimal irradiation positions for both types of irradiations. However, the presented results should be treated rather as a preliminary assessment of the irradiation environment. Detailed calculations should be performed for the fixed specification of the irradiation experiment, which is the next step of the study. In the course of our work, we found that there is a possibility of further improvement through: a) optimization of the irradiation geometry with regard to the sample-source positioning, e.g. introduction of axial thermalization zones,

b) applying a stronger neutron source, which in practice is related to stronger radiation protection and security requirements,

c) increasing the spatial range of observations for reactions on Th by applying more thorium samples in various positions in the assembly.

Acknowledgments. The research was partially supported by PL Grid Infrastructure available at the Academic Computer Centre CYFRONET AGH. In addition, partial financial support for this study under the grant agreement 11.11.210.377 by the Polish Ministry of Science and Higher Education is kindly acknowledged.

\section{References}

1. International Atomic Energy Agency. (2012). Role of thorium to supplement fuel cycles of future nuclear energy systems. Vienna: IAEA. (Nuclear Energy Series No. NF-T-2.4).

2. Serfontein, D. E., \& Mulder, E. J. (2014). Thorium-based fuel cycles: Reassessment of fuel economics and proliferation risk. Nucl. Eng. Des., 271, 106-113.

3. Vijayan, P., Shivakumar, V., Basu, S., \& Sinha, R. (2017). Role of thorium in the Indian nuclear power programme. Prog. Nucl. Energy, 101(Pt A), 43-52.

4. Abdel-Khalik, S. I., Haldy, P. A., \& Kumar, A. (1984). Blanket design and calculated performance for the Lotus Fusion-Fission Hybrid Experimental Devices Test Facility. Fusion Sci. Technol., 2, 189-208.

5. Bhabha Atomic Research Centre. [access: 10.11.2017], www.barc.gov.in/randd/index.html.

6. Oettingen, M., Cetnar, J., \& Mirowski, T. (2015). The MCB code for numerical modeling of fourth generation nuclear reactors. Computer Sci., 16(4), 329-350.

7. X-5 Monte Carlo Team. (2005). MCNP - A General Monte Carlo N-Particle Transport Code, Version 5. LANL. (Report LA-UR-03-1987).

8. Cetnar, J. (2006). Solution of Bateman equations for nuclear transmutations. Ann. Nucl. Energy, 33, 640-645.

9. McConn, R. J. Jr, Gesh, C. J., Pagh, R. T., Rucker, R. A., \& Williams III, R. G. (2011). Radiation portal monitor project, compendium of material composition data for radiation transport modeling. Revision 1. Pacific Northwest National Laboratory. (PIET-43741TM-963, PNNL-15870).

10. Morss, L. R., Edelstein, N. M., \& Fuger, J. (2010). The chemistry of the actinide and transactinide elements (4th ed.). Dordrecht: Springer.

11. ACK Cyfronet AGH. [access: 10.11.2017], KDM. www.cyfronet.krakow.pl/portal/Prometheus.

12. Martin, R. C., Knauer, J. B., \& Balo, P. A. (2000). Production, distribution and applications of californium-252 neutron sources. Appl. Radiat. Isot., 53, 785-792.

13. Liu, Z., Yang, C., Yang, Y., Zheng, L., \& Rong, L. (2018). Measurement and analysis of ${ }^{232} \mathrm{Th}(\mathrm{n}, 2 \mathrm{n})^{231} \mathrm{Th}$ reaction rate in the thorium oxide cylinder with a D-T neutron. Ann. Nucl. Energy, 111, 660-665.

14. Mohapatra, D. K., Singh, S. S., Riyas, A., \& Mohanakrishnan, P. (2013). Physics aspects of metal fuelled fast reactors with thorium blanket. Nucl. Eng. Des., 265, 1232-1237.

15. Kooyman, T., \& Buiron, L. (2016). Neutronic and fuel cycle comparison of uranium and thorium as matrix for minor actinides bearing-blankets. Ann. Nucl. Energy, 92, 61-71. 